\title{
Theater interkulturell von Klaus Hoffmann und Rainer Klose
}

\section{Birgit Oelschläger}

Die 2008 von der Bundesarbeitsgemeinschaft Spiel und Theater im SchibriVerlag herausgegebene Publikation „Theater interkulturell - Theaterarbeit mit Kindern und Jugendlichen "1 beruht auf den Ergebnissen einer vom Bundesministerium für Bildung und Forschung geförderten bundesweiten Bestandsaufnahme zur theaterpädagogischen Arbeit mit Kindern und Jugendlichen mit Migrationshintergrund. Sie richtet sich an das theaterpädagogische Fachpublikum und möchte neben der Darstellung der aktuellen Situation von Theaterprojekten mit jungen Migranten und Migrantinnen vor allem eine fundierte Diskussion über Konzepte für diese spezifische theaterpädagogische Arbeit anregen und damit zukünftige Projekte politisch, inhaltlich und künstlerisch unterstützen.

So findet sich im Buch nach der ausführlichen Darstellung der empirischen Untersuchungsergebnisse eine Aufsatzsammlung mit Handlungsempfehlungen für zukünftige Theaterprojekte mit Migranten, die auf den Ergebnissen der Studie beruhen sowie Beiträge zur interkulturellen Theaterpädagogik, die zum größten Teil Praxisberichte der Spielleiter/-innen beinhalten. In einem abschließenden Serviceteil befinden sich hilfreiche Literaturempfehlungen, Adressen von Theaterverbänden, Institutionen und Festivals sowie Links und Hinweise zu finanziellen Fördermöglichkeiten.

Die erste bundesweite Bestandsaufnahme basiert auf der Auswertung einer computergestützten schriftlichen Befragung an Hand von Fragebögen, die von knapp 500 Spielleiterinnen im schulischen und außerschulischen Bereich hinsichtlich ihrer Erfahrungen mit 700 Theaterprojekten ausgefüllt wurden. Abgefragt wurden an Hand von 83 Fragen sowohl Aspekte zu den Projektleitern und -leiterinnen wie u.a. zu deren beruflichem Hintergrund und eigenem Migrationshintergrund als auch zu den Theaterprojekten wie u.a. Teilnehmerzusammensetzung und -hintergrund, Arbeitsansätze und Inhalte, Rahmenbedingungen, Wirkungen der Theaterarbeit auf die Teilnehmer/-innen sowie Aufführungen und Kooperationspartner. Die wichtigsten Ergebnisse der Studie seien hier kurz genannt: Die Mehrheit der Projektleiter/-innen verfügt über eine theaterpädagogische Qualifikation, ein Viertel von ihnen über einen eigenen Migrationshintergrund. Die am häufigsten genannten

1 Theater interkulturell. Theaterarbeit mit Kindern und Jugendlichen. Hg. Klaus Hoffmann, Rainer Klose/Bundesarbeitsgemeinschaft Spiel \& Theater e.V. Schibri-Verlag Berlin/Milow/Strasburg 2008 
Trägerinstitutionen sind Schulen und Theater. Was die Teilnehmer/-innen betrifft, stammt der überwiegende Teil aus Schulen, davon die Hälfte aus Gymnasien. Bei der Arbeitsweise liegt der Schwerpunkt auf der Improvisation und Stückentwicklung. An erster Stelle der genannten Stückinhalte rangiert die Familie. Die Theaterprojekte erhalten zu 71\% öffentliche Gelder und 42\% haben an Theaterfestivals teilgenommen.

Während die vorangegangen Ergebnisse auch ein Abbild theaterpädagogischer Arbeit in der Bundesrepublik generell liefern, so erscheint spezifischer, dass bei den behandelten Stückinhalten die Familienbeziehungen mit 53\% an erster Stelle stehen, 70\% der Spielleiter/-innen die multiethnische Gruppenzusammensetzung als positiv für den künstlerischen Prozess bewerten und $84 \%$ meinen, durch die Theaterarbeit hätten sich die Integrationschancen der Teilnehmer/-innen deutlich verbessert. Bezüglich der Förderung von Integrationsfähigkeit durch Theaterspielen fassen Klaus Hoffmann und Rainer Klose in ihren „Handlungsempfehlungen“ zusammen:

In unserer Bestandsaufnahme wird von den Projektleiter/-innen der Gewinn an individuellen Kompetenzen als Schlüsselqualifikation für die Lebenswelt, eben auch für die Integrationsfähigkeit, betont: Selbstbewusstsein und Kommunikationsfähigkeit, selbständig werden und Vertrauen in die eigenen Fähigkeiten bekommen. Auch die heterogene Zusammensetzung der Gruppen aus verschiedenen Kulturen und Religionen bekommt eine zentrale Bedeutung und eigene Dynamik: Offenheit, Neugier, Toleranz und Verständnis. Bereitschaft, sich mit anderen Kulturen auseinander zu setzen und Unterschiede zu respektieren, sind wichtige Fähigkeiten für gelingende Integration, die nicht als ein von der Mehrheitskultur geforderter Zustand begriffen wird, sondern als vielgestalterischer Prozess mit individuellen Wahlmöglichkeiten. ${ }^{2}$

In diesem zentralen Artikel machen die Autoren deutlich, wie wichtig es ist, dass Deutschland als modernes Einwanderungsland auf den gestiegenen Anteil junger Migranten und Migrantinnen besonders durch die Förderung kultureller Bildung reagiert. ${ }^{3}$

Desweiteren werden Handlungsempfehlungen ausgesprochen, die nicht nur allgemein das Bewusstsein für interkulturelle Prozesse schärfen sollen, sondern auch konkrete bildungspolitische Konsequenzen einfordern.

Die hier besprochene Publikation versucht einen Grundstein in Bezug auf ein theaterpädagogisches Konzept zu legen, das sowohl integrationspädagogische als auch künstlerische Ansätze des interkulturellen Theaters vereint. Durch die verschiedenen Blickwinkel der Autoren wird in den Aufsätzen der Spagat zwischen Integration und interkulturellem Dialog deutlich, denn bei den hier als ,junge Migranten und Migrantinnen“bezeichneten Schülern und Schülerinnen

\footnotetext{
${ }^{2}$ Hoffmann/Klose: Handlungsempfehlungen zur Theaterarbeit mit jungen Migranten/innen, a.a.O. S. 81

3 vgl. dazu auch das Grußwort von Annette Schavan, a.a.O., wo sie auf den 2007 vorgelegten Nationalen Integrationsplan verweist und dessen zentralem Anliegen der Vermittlung kultureller Bildung
} 
handelt es sich größtenteils um die sogenannte zweite und dritte Generation von Migrantenfamilien. Eine Fragestellung, die das Buch auch aufwirft, lautet daher, was macht diese spezifische kulturelle Identität aus und wie kann dieser Hintergrund inhaltlich, pädagogisch und ästhetisch für das Theater nutzbar gemacht werden. Barbara Kantel schreibt, dass „die Beziehungsgeflechte und der Alltag vieler Jugendlicher sehr vom kulturellen Hintergrund ihrer Familien geprägt sind“ und dass das gewählte Stückthema „Heimat“ in ihrer Produktion am Staatstheater Hannover dem Bedürfnis der Jugendlichen entsprach, sich dabei mit der eigenen Herkunft auseinanderzusetzen.

Miriam Tscholl dagegen betont in ihrem Praxisbericht über eine Produktion mit jungen Migranten am Staatstheater Hannover, dass „sie (die Teilnehmer) ihr Theater als Kunstform begreifen wollten, dafür eine ernstgemeinte öffentliche Anerkennung bekommen (wollten) und sich nicht als gutgemeinte Integrationsmaßnahme präsentieren (wollten).“

Auf den letzten Aufsatz im Buch sei noch besonders verwiesen, da Romi Domkowsky darin erste Ergebnisse ihrer Studie zur Wirkung des Theaterspielens auf junge Menschen auswertet und dabei die Förderung differenzierter personaler Kompetenzen durch Theaterspielen deutlich wird. ${ }^{4}$

Dass die Theaterpädagogik jedoch nicht nur inhaltlich-thematische sowie integrationsfördernde und persönlichkeitsstärkende, sondern auch ästhetische Konsequenzen aus einem interkulturellen Ansatz in der Theaterarbeit mit jungen Migranten ziehen könnte, zeigt Wolfgang Sting in seinem Artikel über interkulturelles Theater und Theaterpädagogik, wenn er auf „eine Diskrepanz zwischen der thematischen Aktualität interkultureller Fragen und dem künstlerischen Interesse an einer theaterästhetischen Umsetzung" in Deutschland verweist. ${ }^{5}$

Der Publikation ist es damit gelungen, das gesamte Spektrum von Möglichkeiten einer interkulturellen Theaterpädagogik aufzuzeigen und zugleich einen Einblick sowohl in den theoretischen Diskurs als auch in die Praxis der Spielleiter/-innen zu gewähren.

\section{Bibliographie}

Klaus Hoffmann/Rainer Klose (Hrsg.) (2008): Theater interkulturell:

Theaterarbeit mit Kindern und Jugendlichen, Berlin/Milow/Strasburg: Schibri Verlag

Bestelladresse: BAG Spiel \& Theater, Simrockstr. 8, 30171 Hannover, Tel. 0511.458 17 99, FAX 0511.45831 05, E-Mail: info@bag-online.de, Internet: www.bag-online.de

\footnotetext{
${ }^{4}$ Romi Domkowsky: Wie Theaterspielen die persönliche Entwicklung fördern kann. Erste Ergebnisse einer Längsschnittuntersuchung, a.a.O. S. $158 \mathrm{ff}$

${ }^{5}$ Wolfgang Sting: Anderes sehen. Interkulturelles Theater und Theaterpädagogik, a.a.O. S. 102
} 